\title{
Robust and risk-averse routing algorithms in road networks
}

\author{
E. Crisostomi $*$, S. Kirkland ${ }^{* *}$, R. Shorten ${ }^{* *}$ \\ * Department of Energy and Systems Engineering, \\ University of Pisa, Largo Lucio Lazzarino 1, 56127 Pisa, Italy \\ e-mail: emanuele.crisostomi@gmail.com \\ ** Hamilton Institute, \\ National University of Ireland - Maynooth (NUIM), Co. Kildare, \\ Ireland \\ e-mail: \{stephen.kirkland, robert.shorten\}@nuim.ie.
}

\begin{abstract}
Recent works have shown that Markov chain theory can be used to model and realistically describe road network dynamics. Properties of the Markov transition matrix such as the Perron eigenvector, the second eigenvalue, and the Kemeny constant have nice corresponding interpretations in the road network counterparts. Our objective in this paper is to use this modelling paradigm to develop new traffic management applications. In particular, our framework is particularly suited to routing algorithm development, offering new approaches to this old problem. This is the focus of the present paper.
\end{abstract}

Keywords: Markov Chains, Routing algorithms.

\section{INTRODUCTION}

The Google-like model introduced in [Crisostomi et al. (2011)] offers a new paradigm to describe road network dynamics starting from a strong mathematical background based on Markov chain theory. This paper further exploits the same Markov chain framework to derive new tools to support and improve road transport, specifically new routing strategies.

Smart routing of traffic is seen as a major enabler of reduced carbon transport, reduced congestion, and as a tool to increase the range of electric vehicles. It enables a more efficient use of the road network, and it can be used proactively to avoid pollution peaks in certain urban areas. Nowadays a lot of road information is collected in large databases, and optimal paths can be computed in less than one second even for very large distances, for instance using the popular tool Google Maps. Path optimality usually means "minimum travel time". Other common cost functions include shortest path or minimum toll charges. Usually routing service providers suggest three or more alternative paths which are almost equivalent in terms of travel time, and the user takes the final decision on the basis of his experience. For instance, the driver might decide to take the path he is most familiar with, or the road which is most robust with respect to unexpected events (e.g. traffic, car accidents, road works...). This paper shows that such popular ways of individually choosing routing strategies can be formulated in a precise mathematical manner, and solved analytically using the Markov-chain based road network model that was proposed in [Crisostomi et al. (2011)].
Our paper is organised as follows: the next section briefly motivates the benefits of these new routing strategies. Section 3 summarises the Markov chain model proposed in [Crisostomi et al. (2011)] to describe road networks dynamics. Section 4 reviews the concepts of Mean First Passage Times and Kemeny constant from Markov chain theory. Finally, in Section 5 we illustrate the two routing algorithms and this constitutes the main contribution of this paper.

\section{PREAMBLE}

The most popular way to plan a route is to compute the shortest path between a pair of origin/destination roads. Shortest path search algorithms are well established in the literature, and classic approaches include Bellman's routing solutions [Bellman (1958)], Dijkstra's algorithm [Dijkstra (1959)] and algorithms of dynamic programming [Bertsekas (1993)]. Alternative familiar "optimal" paths minimise travel times and toll charges. Several routing service providers, like the popular Google Maps, can be used to compute road-by-road instructions to reach the final destination in minimum time.

It is common experience however that the driver does not always follow the minimum time path suggested by the routing service provider: for instance, the driver might prefer to take a longer path which goes through a known territory rather than taking new roads he is not familiar with. In other circumstances, even if real-time information could guarantee the driver that the fastest path is currently free from traffic, still he might prefer to take another "less optimal" route, as he knows from experience that traffic might increase suddenly. Finally, it is very typical 
that the routing service provider suggests several different routes which require almost the same travel time and the user has no clues to decide which one should be chosen.

In this paper new routing strategies are proposed to support the minimum time optimal solutions and discriminate among a set of similar routes. The proposed routing algorithms are called robust and risk-averse routing respectively and mathematically replicate common-sense based strategies that are a feature of driver behaviour.

By robust routing it is intended that the minimum time path is computed taking into account the probability that the driver might make a mistake at crucial junctions, and therefore unintentionally significantly increase the travel time. This robustness, which mitigates against such effects, is closely related to the driver's familiarity with the road network. Namely, if we know the road network very well, then the probability of making mistakes can be assumed to be zero, and the suggested path coincides with the time optimal path. If we find ourselves in a new environment, we can arbitrarily tune the probability of making a mistake based on our confidence and experience, and the robust optimal path is computed accordingly. We show that such an optimal path is in principle different as the probability of making a mistake changes.

We also propose a second risk-averse routing strategy which prioritises the choice of routes that contain detours from the optimal path. The motivation is that redundancy makes it more likely to find an alternative way to reach the desired destination, should any unexpected event take place (road works, car accident, unexpected traffic...).

The performances and the benefits of the proposed strategies are illustrated through several examples and are validated using the mobility simulator SUMO (Simulation of Urban MObility) [Krajzewicz et al. (2006)]. SUMO is an open source, highly portable microscopic road traffic simulation package that was developed at the Institute of Transportation Systems at the German Aerospace Center, and is licensed under the GPL.

The paper is devoted to the analysis of routing algorithms for road networks, but it will be clear that several of the proposed ideas can be applied as well to design optimal paths in completely different networks for other applications; for example for electric vehicles.

\section{MARKOV CHAIN REPRESENTATION OF A ROAD NETWORK}

Following the approach of [Crisostomi et al. (2011)] we assume that a road network is represented as a directed graph whose nodes correspond to road segments connecting two junctions. Road segments have to be considered with their direction of motion (i.e. road $\mathrm{AB}$ from junction $A$ to $B$ is different from road BA, from junction B to A). If at the end of a road it is possible to take directly a second road then the two corresponding nodes are connected through an edge directed from the first to the second road. This kind of road network is known in literature as a dual network, in contrast to the primal network where nodes correspond to junctions. An example of a very simple dual road network is shown in Figure 1.

In a Markov chain all the information is packed inside

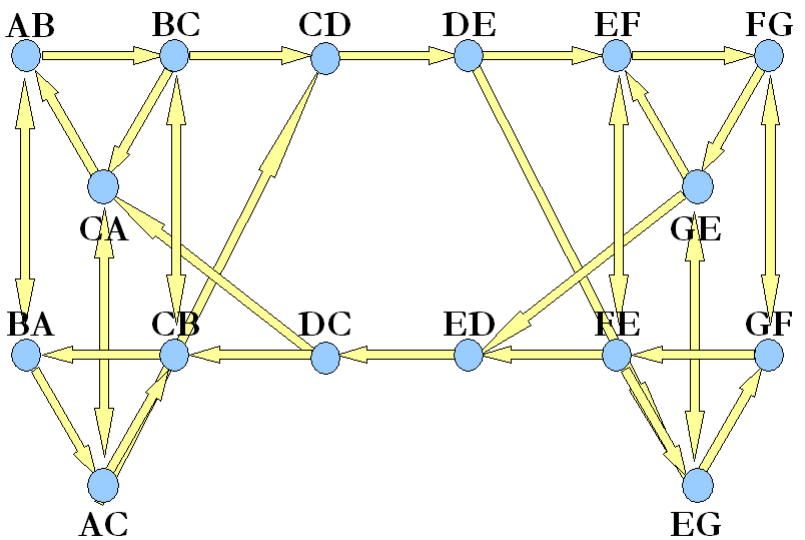

Fig. 1. Example of a road network. For instance, a possible path to go from road CD to DC (which is the same road, opposite way) is to take roads DE, EF, FE, ED.

the transition matrix $\mathbb{P}$, which is filled like in (1), for the example shown in Figure 1.

$$
\mathbb{P}=\left[\begin{array}{ccccc}
\mathbf{P}_{\mathbf{A B} \rightarrow \mathbf{A B}} & P_{A B \rightarrow A C} & P_{A B \rightarrow B A} & \ldots & P_{A B \rightarrow G F} \\
P_{A C \rightarrow A B} & \mathbf{P}_{\mathbf{A C} \rightarrow \mathbf{A C}} & P_{A C \rightarrow B A} & \ldots & P_{A C \rightarrow G F} \\
P_{B A \rightarrow A B} & P_{B A \rightarrow A C} & \mathbf{P}_{\mathbf{B A} \rightarrow \mathbf{B A}} & \ldots & P_{B A \rightarrow G F} \\
\vdots & \vdots & \vdots & \ddots & \vdots \\
P_{G F \rightarrow A B} & P_{G F \rightarrow A C} & P_{G F \rightarrow B A} & \ldots & \mathbf{P}_{\mathbf{G F} \rightarrow \mathbf{G F}}
\end{array}\right]
$$

The diagonal elements $P_{j \rightarrow j}$ are related to the average travel time $t t_{j}$ required to drive along the particular road $j$, and once travel times are known (e.g. because they are measured from traffic data) they can be easily computed as

$$
P_{j \rightarrow j}=\frac{t t_{j}-1}{t t_{j}}, \quad j=1, \ldots, n .
$$

According to equation (2) the average time required to leave the state $j$ in the Markov chain is exactly equal to $t t_{j}$, as proved in [Crisostomi et al. (2011)]. The off-diagonal elements $P_{i \rightarrow j}$ of the transition matrix are obtained as $\left(1-P_{i \rightarrow i}\right) \cdot P_{i j}$, where $P_{i j}$ is the observed probability that at the end of road $i$ a car will turn into road $j$. If nodes $i$ and $j$ are not connected, this probability is zero.

The transition matrix (1) can be constructed after observing average travel times and junction turning probabilities. This information can be easily gathered by exploiting cars as mobile sensors and collecting average data. In this paper the same data is gathered from simulating traffic in the road network of interest, using the software SUMO.

\section{ROAD NETWORKS MARKOV CHAINS}

\subsection{Perron eigenvector}

Transition matrices corresponding to road networks are irreducible because the associated graph is strongly connected, which is implied by the fact that from an arbitrary starting point it is always possible to reach any destination. 
The left-hand Perron eigenvector $\pi$ is the unique vector defined by $\pi^{T} \mathbb{P}=\pi^{T}$, such that $\pi>0,\|\pi\|_{1}=1$. Except for positive multiples of $\pi$ there are no other non-negative left eigenvectors for $\mathbb{P}$. By saying $\pi>0$ it is meant that all entries of vector $\pi$ are strictly positive.

One of the main properties of irreducible Markov chains is that the $i^{t h}$ component $\pi_{i}$ of vector $\pi$ represents the long-run fraction of time that the chain will be in state $S_{i}$. The row vector $\pi^{T}$ is also called the stationary distribution vector of the Markov chain.

In road networks the entry of the stationary distribution vector is related to the density of cars along the corresponding road [Crisostomi et al. (2011)].

\subsection{Mean First Passage Time Matrix}

A transition matrix $\mathbb{P}$ with 1 as a simple eigenvalue gives rise to a singular matrix $\boldsymbol{I}-\mathbb{P}$ (where the identity matrix $\boldsymbol{I}$ has appropriate dimensions) which is known to have a group inverse $Q^{\#}=(\boldsymbol{I}-\mathbb{P})^{\#}$. The group inverse is the unique matrix such that $(\boldsymbol{I}-\mathbb{P})(\boldsymbol{I}-\mathbb{P})^{\#}=$ $(\boldsymbol{I}-\mathbb{P})^{\#}(\boldsymbol{I}-\mathbb{P}),(\boldsymbol{I}-\mathbb{P})(\boldsymbol{I}-\mathbb{P})^{\#}(\boldsymbol{I}-\mathbb{P})=(\boldsymbol{I}-\mathbb{P})$, and $(\boldsymbol{I}-\mathbb{P})^{\#}(\boldsymbol{I}-\mathbb{P})(\boldsymbol{I}-\mathbb{P})^{\#}=(\boldsymbol{I}-\mathbb{P})^{\#}$. More properties of group inverses and their applications to Markov chains can be found in [Meyer (1975)].

The mean first passage time $m_{i j}$ from the state $S_{i}$ to state $S_{j}$ is the expected number of steps to arrive at destination $S_{j}$ when the origin is $S_{i}$. If we denote as $q_{i j}^{\#}$ the entries of the matrix $Q^{\#}$, then the mean first passage times can be computed easily according to (see [Cho and Meyer (2001)] for example)

$$
m_{i j}=\frac{q_{j j}^{\#}-q_{i j}^{\#}}{\pi_{j}}, \quad i \neq j,
$$

where it is intended that $m_{i i}=0, \quad i=1, \ldots, n$.

\subsection{Kemeny constant}

The Kemeny constant is defined as

$$
K=\sum_{j=1}^{n} m_{i j} \pi_{j},
$$

where the right hand side is surprisingly independent of the choice of $i$ [Kemeny and Snell (1960)]. An interpretation of this result is that the expected time to get from an origin state $S_{i}$ to a destination state $S_{j}$ selected randomly according to the equilibrium measure $\pi$ does not depend on the starting point $S_{i}$ [Doyle (2010)]. Therefore the Kemeny constant is an intrinsic quantity of a Markov chain, and if the transition matrix $\mathbb{P}$ has eigenvalues $\lambda_{1}=1, \lambda_{2}, \ldots, \lambda_{n}$ then another way of computing $K$ is [Levene and Loizou (2002)]

$$
K=\sum_{j=2}^{n} \frac{1}{1-\lambda_{j}} .
$$

Equation (5) emphasises the fact that $K$ is only related to the particular matrix $\mathbb{P}$ and that it increases if $\mathbb{P}$ has a real eigenvalue close to 1 .

\section{ROUTING ALGORITHMS}

\subsection{Minimum time path}

A required assumption to construct the Markov chain transition matrix is the knowledge of average travel times, therefore this knowledge can be exploited to design optimal routing strategies.

Given a pair of origin/destination roads the minimum time path can be computed according to the well known Dijkstra's algorithm. As travel times change according to traffic conditions, it is possible to recompute optimal paths every time the Markov chain transition matrix is updated with the latest available information. In principle optimal routing strategies are time variant [Tian et al. (2009)]. In the next sections we show how properties of Markov chains, namely mean first passage times and the Kemeny constant can be used as additional routing tools to further support the driver in his path choice.

\subsection{Robust minimum time path}

Random walk optimality: $\quad$ Let us consider a random walk in the road network: let $A$ be the adjacency matrix of the road network, i.e. the $(0,1)$ matrix containing ones in position $A_{i j}$ if the $i^{\text {th }}$ road is directly connected to the $j^{\text {th }}$ road. All other entries, including those along the main diagonal, are zeros. Let us consider that out-degree matrix $D_{\text {out }}$ which is a diagonal matrix where the entry $D_{i i}=\sum_{i=1}^{n} A_{i j}$ indicates the number of roads directly connected to road $i$. Then a random walk on the road network is described by the transition matrix

$$
\mathbb{P}_{R W}=D_{\text {out }}^{-1} A \text {. }
$$

The mean first passage time matrix computed on the Markov chain described by $\mathbb{P}_{R W}$ can be interpreted as the expected time to go from each possible origin to destination if the motion is completely random (i.e. at the end of a road segment, the next one is chosen randomly).

Robustifying the minimum time solution: Let us assume now that the minimum time path is known (e.g. it is given by a path search service provider, like GoogleMaps), and let us call $i_{1} i_{2}, \ldots, i_{m-1} i_{m}$ the optimal sequence of roads from the origin road $i_{1}$ to the destination road $i_{m}$ (for instance here we assume that the optimal path is composed of $m$ roads). Then let us change the random walk matrix $\mathbb{P}_{R W}$ so that if the connection between roads $i_{j}, i_{k}$ appears in the optimal sequence, then $\mathbb{P}_{R W}\left(i_{j}, i_{k}\right)$ (i.e. the entry in row $i_{j}$ and column $i_{j}$ ) is $1-\epsilon$ and all other entries in the same row different from zero are given the same probability, which is $\epsilon /\left(D_{\text {out }}\left(i_{j}, i_{j}\right)-1\right)$ so that the matrix remains row stochastic. If $i_{k}$ is the only candidate successive road of $i_{j}$ (i.e. $D_{\text {out }}\left(i_{j}, i_{k}\right)=1$ ), then the row does not get modified. The parameter $\epsilon \in(0,1)$ represent the probability of taking a wrong turn at a junction. The parameter can be tuned appropriately as a function of the driver's ability and knowledge of the area of interest.

Let us denote as $\mathbb{P}_{r o b}^{o p t}$ the modified transition matrix, then let us compute the associated mean first passage time matrix $M_{\text {rob }}^{o p t}$ according to equation (3). Then the expected time to get from $i_{1}$ to $i_{m}$ is given by the entry $M_{r o b}^{o p t}\left(i_{1}, i_{m}\right)$ and is a function of $\epsilon$. 
Remark 1. The optimal mean first passage time solution tends to the minimum time path as $\epsilon$ tends to zero, i.e. as errors become very unlikely. Yet, if $\epsilon$ is exactly zero, connectivity of the graph may be lost (i.e. the corresponding Markov chain is not irreducible anymore) and the mean first passage times matrix is not well defined.

\section{Example}

A short example is provided to illustrate the different routing algorithms shown so far, and compare the corresponding performances. Let us consider the simple road graph shown in Figure 2. According to the procedure de-

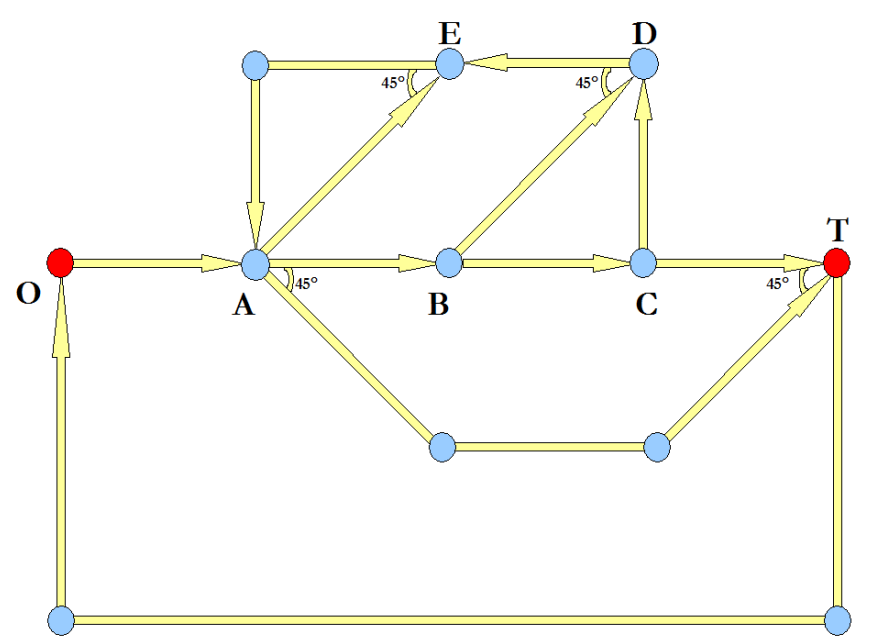

Fig. 2. Example of a primal network. The objective is to compute the best path to go from the origin road $O$ to the target road $T$.

scribed in [Crisostomi et al. (2011)] the first step consists in transforming the primal network shown in Figure 2 in the dual one, where nodes correspond to roads. Let us name the roads after the junctions they connect (i.e. the road from junction $B$ to $C$ is named $B C$ ). For simplicity, we assume that travel times are proportional to road lengths, and are normalised so that road $B C$ takes for instance one unit of time, while $B D$ requires $\sqrt{2}$ time units. The random walk transition matrix $\mathbb{P}_{R W}$ associated to the dual network has the shape (1), its diagonal entries are computed according to (2) and the off-diagonal entries are given the same probability (6). The transition matrix $\mathbb{P}_{R W}$ is now reported for clarity; rows (and columns) of such a matrix correspond to the starting (arriving) road in the dual network of Figure 2, namely they are $O A, A B, B C$, $C T, A T, T O, E A, A E, D E, B D$, and $C D$.

$$
\mathbb{P}_{R W}=\left[\begin{array}{ccccccccccc}
0 & 1 / 3 & 0 & 0 & 1 / 3 & 0 & 0 & 1 / 3 & 0 & 0 & 0 \\
0 & 0 & 1 / 2 & 0 & 0 & 0 & 0 & 0 & 0 & 1 / 2 & 0 \\
0 & 0 & 0 & 1 / 2 & 0 & 0 & 0 & 0 & 0 & 0 & 1 / 2 \\
0 & 0 & 0 & 0 & 0 & 1 & 0 & 0 & 0 & 0 & 0 \\
0 & 0 & 0 & 0 & 0.74 & 0.26 & 0 & 0 & 0 & 0 & 0 \\
0.13 & 0 & 0 & 0 & 0 & 0.87 & 0 & 0 & 0 & 0 & 0 \\
0 & 1 / 6 & 0 & 0 & 1 / 6 & 0 & 1 / 2 & 1 / 6 & 0 & 0 & 0 \\
0 & 0 & 0 & 0 & 0 & 0 & 0.71 & 0.29 & 0 & 0 & 0 \\
0 & 0 & 0 & 0 & 0 & 0 & 1 & 0 & 0 & 0 & 0 \\
0 & 0 & 0 & 0 & 0 & 0 & 0 & 0 & 0.71 & 0.29 & 0 \\
0 & 0 & 0 & 0 & 0 & 0 & 0 & 0 & 1 & 0 & 0
\end{array}\right]
$$

\begin{tabular}{|c|c|c|}
\hline Parameter $\epsilon$ & Optimal path & Alternative path \\
\hline$\epsilon=0$ & 4 & 4.828 \\
\hline$\epsilon=0.05$ & 4.9307 & 5.2733 \\
\hline$\epsilon=0.1$ & 5.8355 & 5.7181 \\
\hline$\epsilon=0.2$ & 7.5456 & 6.6078 \\
\hline
\end{tabular}

Table 1. Comparison between the minimum time path and an alternative path as a function of the probability of error $\epsilon$.

Dijkstra's algorithm can be used to compute the minimum time path using the knowledge of travel times, and the optimal path is clearly $O A-A B-B C-C T$ with overall travel time 4 units, while the second best path (we will denote it as alternative path) is $O A-A T$ which takes $2+2 \sqrt{2}$ time units. We now perturb the two optimal paths assuming a probability of making mistakes equal to $\epsilon$, so the perturbed transition matrix associated to the minimum time path becomes

$$
\mathbb{P}_{R W}=\left[\begin{array}{ccccccccccc}
0 & 1-\epsilon & 0 & 0 & \epsilon / 2 & 0 & 0 & \epsilon / 2 & 0 & 0 & 0 \\
0 & 0 & 1-\epsilon & 0 & 0 & 0 & 0 & 0 & 0 & \epsilon & 0 \\
0 & 0 & 0 & 1-\epsilon & 0 & 0 & 0 & 0 & 0 & 0 & \epsilon \\
0 & 0 & 0 & 0 & 0 & 1 & 0 & 0 & 0 & 0 & 0 \\
0 & 0 & 0 & 0 & 0.74 & 0.26 & 0 & 0 & 0 & 0 & 0 \\
0.13 & 0 & 0 & 0 & 0 & 0.87 & 0 & 0 & 0 & 0 & 0 \\
0 & 1 / 6 & 0 & 0 & 1 / 6 & 0 & 1 / 2 & 1 / 6 & 0 & 0 & 0 \\
0 & 0 & 0 & 0 & 0 & 0 & 0.71 & 0.29 & 0 & 0 & 0 \\
0 & 0 & 0 & 0 & 0 & 0 & 1 & 0 & 0 & 0 & 0 \\
0 & 0 & 0 & 0 & 0 & 0 & 0 & 0 & 0.71 & 0.29 & 0 \\
0 & 0 & 0 & 0 & 0 & 0 & 0 & 0 & 1 & 0 & 0
\end{array}\right]
$$

Obviously, diagonal elements are not modified because they are only related to travel times. Similarly it is possible to perturb the transition matrix associated to the alternative path. The obtained transition matrices are used to compute the corresponding mean first passage time matrices and to compare the expected mean first passage times from the origin road to the destination (e.g. entry $(1,6)$ of the mean first passage time matrix). Table 1 compares travel times required by both the paths as a function of the parameter $\epsilon$.

According to the results of Table 1 the alternative path outperforms the optimal path when the probability of making errors increases (in particular the two paths are equally convenient for $\epsilon=8.69 \%$ ). Therefore the robust minimum time path coincides with the optimal path when $\epsilon=0$, while in principle it is different, as in the proposed example, for larger values of $\epsilon$.

\section{Validation of the result}

Simulations were performed to prove the performances of the robust minimum time routing strategy in practice. The same road network of Figure 2 was created in the mobility simulator SUMO [Krajzewicz et al. (2006)]. A flow of cars was designed from road $O A$ to road $T O$ and average travel times were computed for different values of junction turning probabilities as a function of $\epsilon$. Simulations showed that the flow of cars choosing the alternative path required a shorter time to reach their 
destination than the flow of cars choosing the optimal path for both values of $\epsilon=10 \%$ and $20 \%$.

\subsection{Risk-averse path}

The risk of a path is computed by summing the criticality of the single roads that compose the path. Network theory provides plenty of indicators that measure the importance of a node. A typical approach follows the idea of edge removal, see for instance [Newman (2005)], [Freeman (1977)] and [Estrada et al. (2009)], where the original network is compared with a new network where in turn each node is deleted. If the performances of the network noticeably degrade, then the deleted node had an important role in the original network. Most of the proposed works in the literature are for undirected graphs, so in this work we suggest the use of the Kemeny constant which can also be extended to the case of the directed road network. As introduced in section 4.3 the Kemeny constant measures the average time in the network required to reach a road averaged over the popularity of the road. Therefore, if the Kemeny constant dramatically increases when a particular road is deleted, it implies that the deleted road was critical, and it should be avoided in the planned route to prevent increased travel times should an unexpected event occur in the road.

Remark 2. Computation of the Kemeny constant after removing a node is consistent provided that the road network remains connected. Mathematically, this can be checked by computing the rank of the corresponding incidence matrix (see for instance reference [Langville and Meyer (2006)]). We will say that a road is infinitely critical if a particular destination is not reachable anymore when the road is removed (the idea is that infinite time is required to reach it). However in practice road networks are usually redundant and there are several alternative paths to reach the same destination.

In the next section an example is provided to support and illustrate the proposed method.

\section{Example}

We consider the road graph described by the primal network shown in Figure 3. The network is designed in a redundant way, so that each destination is still reachable if an unpredictable event leads to the closure of an arbitrary road. The objective is to find the best path to go from $I$ to $F$. Assuming for simplicity that all roads take the same travel time (i.e. one time unit), then the optimal solutions are the paths $I B-B C-C D-D E-E F, I H-H C-C D-$ $D G-G F, I J-J K-K L-L M-M F, I H-H C-C D-$ $D E-E F$ and $I B-B C-C D-D G-G F$. We compute the criticality of each road by deleting the node and computing the Kemeny constant of the remaining network according to equation (4) and then compare the overall criticality of the candidate optimal paths. Figure 4 shows the criticality of each single road, and we notice that the most critical roads are $I J, M F, F M, G H$ and $J I$ respectively. This is in accordance with the visual inspection of the road network which shows two main cluster of roads that are connected through two two-ways roads $(I J-J I$ and $F M-$ $M F)$. Also $G H$ is an important road, as without it the only connection between the upper left and right groups of roads is through road $D C$. Therefore, as expected,

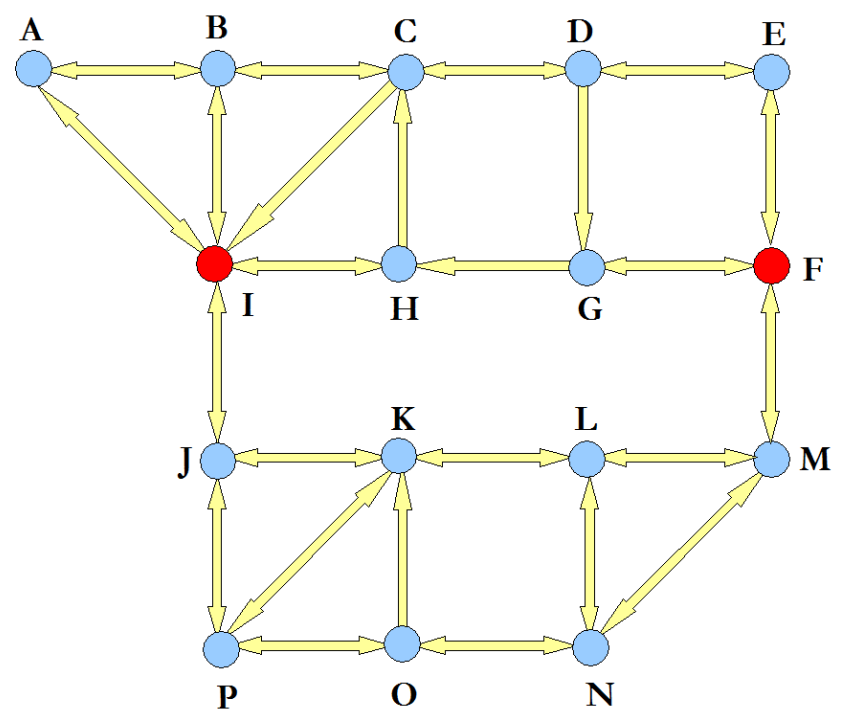

Fig. 3. Example of a redundant primal network. The objective is to compute the best path in terms of riskaversion to go from the origin $I$ to the target $F$.

\begin{tabular}{|c|c|c|}
\hline Candidate Optimal Path & Time required & Overall criticality \\
\hline$I B-B C-C D-D E-E F$ & 5 & 227.73 \\
\hline$I H-H C-C D-D G-G F$ & 5 & 227.07 \\
\hline$I J-J K-K L-L M-M F$ & 5 & 236.52 \\
\hline$I H-H C-C D-D E-E F$ & 5 & 227.53 \\
\hline$I B-B C-C D-D G-G F$ & 5 & 227.27 \\
\hline
\end{tabular}

Table 2. Criticality provides a way of differentiating and classifying otherwise similar paths.

The third path is the least risk-averse.

the Kemeny constant provides an interesting quantitative evaluation of criticality. Table 2 compares the criticality of the 5 candidate optimal paths. According to the criticality approach, path $I H-H C-C D-D G-G F$ is the best one, while $I J-J K-K L-L M-M F$ is clearly the least convenient.

\section{CONCLUSION}

This paper describes one of the possible applications of the new paradigm of modeling road network dynamics described in paper [Crisostomi et al. (2011)], namely routing strategies. Two different strategies, called robust and risk-averse respectively, have been proposed to discriminate between different paths which provide very similar performances in terms of travel times. Current work is focusing in taking into explicit account also the variance of the travel times, which is known to play an important role in routing advisors, and to develop new applications from 


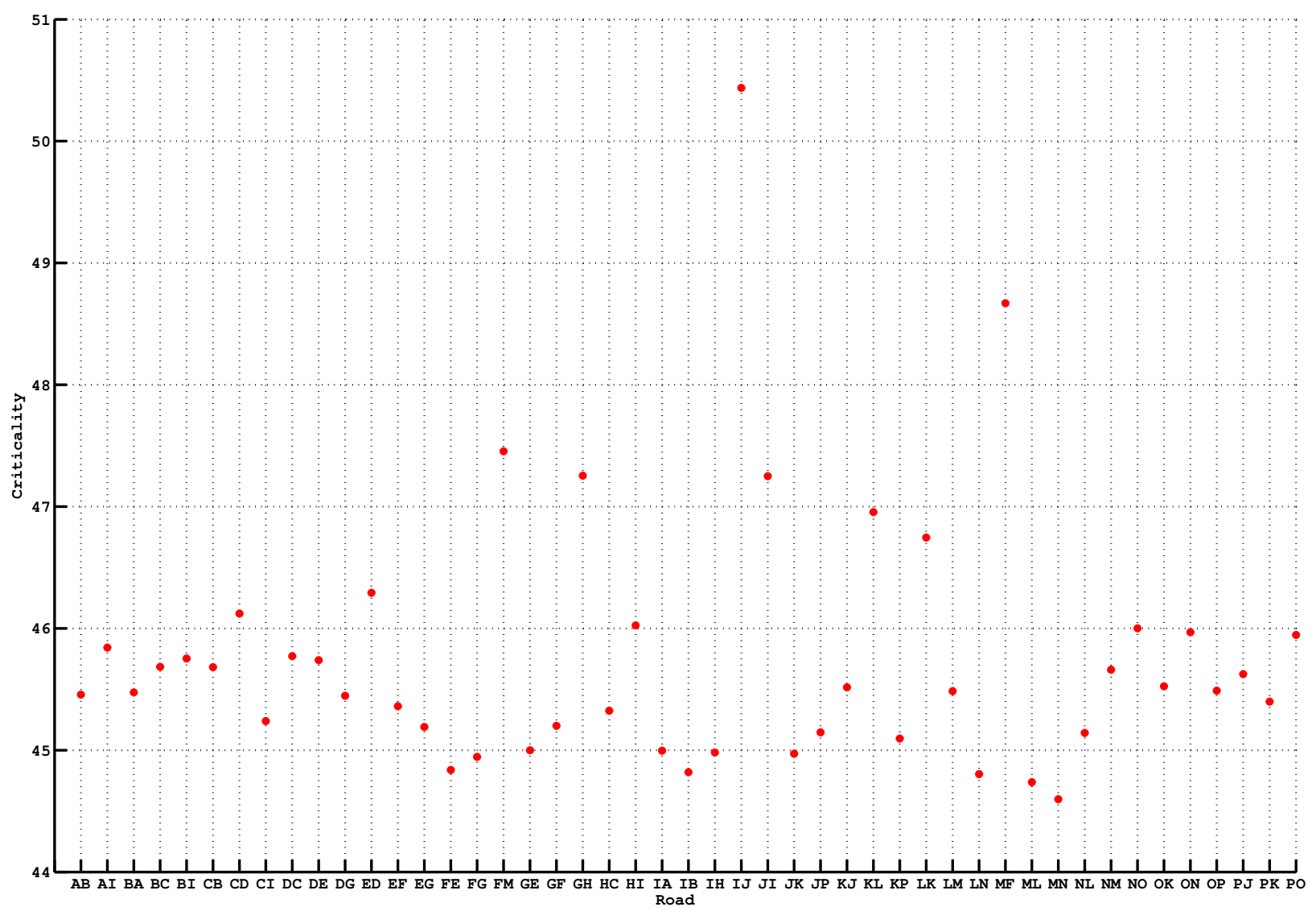

Fig. 4. Importance of every single road is evaluated in terms of criticality. This implies that a road is very important if average travel times increase considerably when the road is closed.

the same paradigm of [Crisostomi et al. (2011)]. Routing strategies for different classes of vehicles (priority routing, minimum emission routing and electric vehicles routing) are also currently investigated.

\section{ACKNOWLEDGEMENTS}

Stephen Kirkland is supported in part by the Science Foundation Ireland under Grant No. SFI/07/SK/I1216b. Robert Shorten and Emanuele Crisostomi are supported in part by Science Foundation Ireland under grant number PI Award 07/IN.1/1901.

\section{REFERENCES}

R. Bellman. On a routing problem. Quarterly of Applied Mathematics, 16:87-50, 1958.

E.W. Dijkstra. A note on two problems in connexion with graphs. Numer. Math., 1:269-271, 1959.

D.P. Bertsekas. A simple and fast label correcting algorithm for shortest paths. Networks, 23:703-709, 1993.

E. Crisostomi, S. Kirkland, and R. Shorten. A Google-like model of road network dynamics and its application to regulation and control. International Journal of Control, accepted for publication, 2011.

D. Krajzewicz, M. Bonert, and P. Wagner. The open source traffic simulation package SUMO. In RoboCup 2006 Infrastructure Simulation Competition, Bremen, Germany, 2006.
C.D. Meyer. The role of group generalized inverse in the theory of finite Markov Chains. SIAM Review, 17:443464, 1975.

G.E. Cho, and C.D. Meyer. Comparison of perturbation bounds for the stationary distribution of a Markov chain. Linear Algebra and its Applications, 335:137-150, 2001.

J.G. Kemeny, and J.L. Snell. Finite Markov Chains. Van Nostrand, Princeton, 1960.

P. Doyle. The Kemeny constant of a Markov chain. Preprint, 2009, available online at URL http://math.dartmouth.edu/ doyle/docs/kc/kc.pdf.

M. Levene, and G. Loizou. Kemeny's constant and the random surfer. American Mathematical Monthly, 109: 741-745, 2002.

Y. Tian, K.C.K. Lee, and W.-C. Lee. Monitoring minimum cost paths on road networks. Proc. of the $17^{\text {th }}$ ACM International Conference on Advances in Geographic Information Systems, 2009.

M.E.J Newman. A measure of betweenness centrality based on random walks. Social Networks, 27:39-54, 2005.

L.C. Freeman. A set of measures of centrality based upon betweenness. Sociometry, 40:35-41, 1977.

E. Estrada, D.J. Higham, and N. Hatano. Communicability betweenness in complex networks. Physica A, 388: 764-774, 2009.

A.N. Langville, and C.D. Meyer. Google's PageRank and beyond - The science of search engine rankings. Princeton University Press, 2006. 